\title{
Prognostic role of derived neutrophil-to- lymphocyte ratio in surgical triple-negative breast cancer
}

This article was published in the following Dove Press journal: Cancer Management and Research

\author{
Kuojun Ren, ${ }^{1, *}$ Yachao \\ Yin, ${ }^{2, *}$ Fang $\mathrm{He},{ }^{3}{ }^{\text {Yi Shao, }}{ }^{4}$ \\ Shengying Wang' \\ 'Department of Head-Neck and \\ Breast Surgery, Anhui Provincial \\ Cancer Hospital, The First Affiliated \\ Hospital of The University of \\ Science and Technology of China \\ (USTC), Division of Life Sciences and \\ Medicine, USTC, Hefei, Anhui, People's \\ Republic of China; ${ }^{2}$ Department of \\ Radiotherapy, Anhui Provincial Cancer \\ Hospital, The First Affiliated Hospital \\ of USTC, Division of Life Sciences and \\ Medicine, USTC, Hefei, Anhui, People's \\ Republic of China; ${ }^{3}$ Department of \\ Respiratory Medicine, Anhui Chest \\ Hospital, Hefei, Anhui, People's \\ Republic of China; ${ }^{4}$ Department of \\ Ophthalmology, The First Affiliated \\ Hospital of Nanchang University, \\ Nanchang, Jiangxi, People's Republic \\ of China \\ *These authors contributed equally to \\ this work
}

Correspondence: Shengying Wang Department of Head-Neck and Breast Surgery, Anhui Provincial Cancer Hospital, The First Affiliated Hospital of The University of Science and Technology of China (USTC), Division of Life Sciences and Medicine, USTC, Hefei, No 107, Huanhu East Road, Shushan District, Hefei, Anhui, 23000I, People's Republic of China

Email Shengywang@163.com

Yi Shao

Department of Ophthalmology, The First Affiliated Hospital of Nanchang University, No 17, Yongwaizheng Street, DongHu District, Nanchang 330006, Jiangxi, People's Republic of China Email freebee99@163.com
Introduction: The role of derived neutrophil-to-lymphocyte ratio (dNLR) in predicting the prognosis of patients with triple-negative breast cancer (TNBC) has not been well studied. Here, we attempted to investigate the significance of dNLR in predicting the prognosis of patients with surgical (nonmetastatic) TNBC.

Methods: A total of 281 patients diagnosed with surgical TNBC in The First Affiliated Hospital of University of Science and Technology of China from February 2005 to March 2015 were retrospectively included in this study. Kaplan-Meier curve analysis was used to assess the disease-free survival (DFS) and overall survival (OS). We used Cox regression model to assess the prognostic significance of pretreatment dNLR and other clinicopathological parameters in TNBC patients.

Results: The median DFS in TNBC patients who had low dNLR and high dNLR was 28.9 and 15.1 months $(P<0.001)$, respectively, whereas the median OS in patients who had low dNLR and high dNLR was 71.2 and 42.3 months $(P<0.001)$, respectively. In patients aged $\leq 50$ years and with invasive ductal carcinoma, a low dNLR predicted better DFS and OS compared with a high dNLR. Multivariate analysis demonstrated that the increased dNLR was a risk factor of poor DFS (HR=1.90, 95\% CI: 1.52-2.46, $P=0.007)$ and OS (HR=2.56, 95\% CI: $1.69-3.58, P=0.001)$.

Conclusion: Pretreatment dNLR is an independent factor of prognosis for TNBC patients, which potentially allows clinical doctors to improve outcomes of patients with high dNLR by treating with aggressive therapy, such as high-dose adjuvant chemotherapy and radiotherapy.

Keywords: dNLR, TNBC, inflammation, immunity, prognosis

\section{Introduction}

Triple-negative breast cancer (TNBC) accounts for about 10\%-20\% of newly diagnosed breast cancer. It is characterized by the negative expression of human EGFR-2, estrogen receptor, and progesterone receptor. ${ }^{1}$ Female patients with TNBC were commonly relapsed and progressed. The peak recurrence rate was observed from the third year to the fifth year after diagnosis. TNBC is featured by intrinsic aggressive tumor pathology, such as high levels of histological grade, proliferation, TP53 mutations, and mitotic index, which leads to larger tumor sizes and poorer clinical outcomes. ${ }^{2,3}$

Therefore, it is particularly important to predict the prognosis of these patients. Currently, prognostic factors for patients with TNBC mainly include histological grade, tumor size, and lymph node status. Several novel prognostic factors such as cfDNA, lymphocyte infiltration, and circulating tumor cells have been identified in recent years. ${ }^{4-7}$ The clinical application of these markers is limited because the cost of detecting these factors is high and there is a lack of evidence regarding their prognostic value. 
Accumulating evidence indicated that systemic inflammation can be a marker for predicting the prognosis of patients with a variety of cancers, for example, breast cancer. ${ }^{8-20}$ Systemic inflammation can be monitored using hematologic or biochemical markers, such as elevated C-reactive protein, leukocyte, neutrophil, platelet cell counts, and hypoalbuminemia. The leukocyte count minus neutrophil count was equivalent to the count of lymphocyte. The derived neutrophil-to-lymphocyte ratio (dNLR) was defined as neutrophil count/(leukocyte count - neutrophil count). Therefore, the high dNLR may be due to the increased neutrophil count or decreased lymphocyte count. Several other studies have used the dNLR (neutrophil/leukocyte - neutrophil) as a prognostic indicator for cancers; their results have shown that elevated dNLR was related to poor prognosis of patients with lung cancer, renal cell carcinoma, pancreatic cancer, gastric cancer, urothelial carcinoma, hepatocellular carcinoma, colorectal cancer, and lymphoma. ${ }^{21-27}$ The evidence for a prognostic role of dNLR in breast cancer is rare and controversial. Thus, we hoped to explore the prognostic role of dNLR in surgical TNBC patients.

\section{Methods}

A total of 281 patients diagnosed with surgical TNBC in The First Affiliated Hospital of The University of Science and Technology of China, from February 2005 to March 2015, were retrospectively included. We included patients with histological confirmation of TNBC; data for differential blood counts were collected prior to anticancer treatment. We excluded patients with inflammatory disease, immune disease, coronary artery disease, and hematological diseases; suffering from an infectious disease within 1 month of enrollment; using anti-inflammatory or immunosuppressive drugs (steroids, azathioprine, antilymphocyte globulin, and rapamycin) prior to enrollment; and with metastatic or inflammatory breast cancer. The ethics committee of The First Affiliated Hospital of The University of Science and Technology of China approved this study, and the written informed consent was not required for individual patient because this study was retrospective and data were anonymous.

All patients underwent radical mastectomy or breastconserving surgery. Patients who underwent neoadjuvant or adjuvant chemotherapy received anthracyclines, cyclophosphamide, and paclitaxel. There were 202 (71.9\%) patients who received chemotherapy with anthracyclines+cycloph osphamide+paclitaxel, whereas 79 (28.1\%) patients who received anthracyclines+paclitaxel. The radiation dose of postoperative radiotherapy was 50-60 Gy/25-30 fractions.
Data for leukocyte count, neutrophil count, lymphocyte count, patients' age, tumor size, lymph node metastasis, lymphovascular invasion, histological grade, proliferative index (Ki-67), and antitumor therapy (eg, surgery, radiotherapy, chemotherapy, and targeted therapy) were collected. The dNLR was defined as neutrophil count/(leukocyte count neutrophil count). We collected the dNLR 1 week before surgery or neoadjuvant chemotherapy.

The follow-up was regularly conducted every 3 months after surgery until death or discontinuation from the study. Ultrasound imaging, computed tomography, MRI, and positron emission computed tomography were used to assess disease status. The contents of follow-up included the extent of disease progression, death, and discontinuation. The deadline for follow-up was March 10, 2018. Overall survival (OS) is defined as the time from pathological diagnosis to death or lost follow-up. Disease-free survival (DFS) time is defined as the time from operation to the first instance of disease recurrence, metastasis, lost follow-up, or death.

\section{Statistical analysis}

We used the Cox regression model for multivariate analysis to identify independent factors for prognosis in TNBC patients. The OS and DFS were evaluated by the Kaplan-Meier method. The log-rank test was used for the comparison of differences in survival between patients from the two groups. Using the receiver operating characteristic curve (ROC) analysis (Figure S1), dNLR (2.6) with the highest area under the curve was selected as the cutoff value between long and short OS. Patients were divided into low dNLR group and high dNLR group by the cutoff point of dNLR. $P<0.05$ was accepted as the statistically significant difference. The SPSS22.0 software (IBM Corporation, Armonk, NY, USA) was used for data analysis.

\section{Results}

Table 1 shows the clinicopathological parameters for patients. A total of $281 \mathrm{TNBC}$ patients were included in the present study. According to the American Joint Committee on Cancer staging system, 39, 150, and 92 cases of patients were at stage I, II, and III of disease, respectively. Among whom, 39 patients had lymphovascular invasion. Forty-six patients received breast-conserving surgery, and 235 patients underwent modified radical mastectomy. There were 34 , 147, and 100 patients with histopathological grade I, II, and III, respectively. One hundred nine patients had low dNLR, and 172 patients had high dNLR. The median dura- 
Table I Clinicopathological parameters of 28 I patients with triple-negative breast cancer

\begin{tabular}{|c|c|c|}
\hline Parameters & $\mathbf{N}=\mathbf{2 8}$ I & $\%$ \\
\hline \multicolumn{3}{|l|}{ Age (years) } \\
\hline$\leq 50$ & 201 & 72 \\
\hline$>50$ & 80 & 28 \\
\hline \multicolumn{3}{|l|}{ Type of surgery } \\
\hline Breast-conserving surgery & 46 & 16 \\
\hline Radical mastectomy & 235 & 84 \\
\hline \multicolumn{3}{|l|}{ Tumor stage } \\
\hline $\mathrm{pTI}$ & 59 & 21 \\
\hline pT2 & 167 & 59 \\
\hline pT3 & 50 & 18 \\
\hline $\mathrm{pT} 4$ & 5 & 2 \\
\hline \multicolumn{3}{|l|}{ Tumor histology } \\
\hline Invasive ductal carcinoma & 223 & 79 \\
\hline Invasive lobular carcinoma & 55 & 20 \\
\hline Others & 3 & 1 \\
\hline \multicolumn{3}{|l|}{ Lymphovascular invasion } \\
\hline Yes & 39 & 14 \\
\hline No & 242 & 86 \\
\hline \multicolumn{3}{|l|}{ Histological grade } \\
\hline I-II & 181 & 64 \\
\hline III & 100 & 36 \\
\hline \multicolumn{3}{|l|}{$\mathrm{Ki}-67$} \\
\hline$\geq 30 \%$ & 121 & 43 \\
\hline$<30 \%$ & 160 & 57 \\
\hline \multicolumn{3}{|l|}{ Lymph node status } \\
\hline $\mathrm{pNO}$ & 118 & 42 \\
\hline $\mathrm{pNI}$ & 96 & 34 \\
\hline $\mathrm{pN} 2$ & 41 & 15 \\
\hline $\mathrm{pN} 3$ & 26 & 9 \\
\hline \multicolumn{3}{|l|}{ AJCC stage } \\
\hline 1 & 39 & 14 \\
\hline II & 150 & 54 \\
\hline III & 92 & 33 \\
\hline \multicolumn{3}{|l|}{ Adjuvant radiotherapy } \\
\hline Yes & 180 & 64 \\
\hline No & 101 & 36 \\
\hline \multicolumn{3}{|l|}{ Chemotherapy } \\
\hline Neoadjuvant chemotherapy & 59 & 21 \\
\hline Adjuvant chemotherapy & 222 & 79 \\
\hline \multicolumn{3}{|l|}{$\mathrm{dNLR}$} \\
\hline$\geq 2.6$ & 109 & 39 \\
\hline$<2.6$ & 172 & 61 \\
\hline
\end{tabular}

Abbreviations: AJCC, American Joint Committee on Cancer; dNLR, derived neutrophil-to-lymphocyte ratio.

tion of follow-up was 67 months (16-148 months). At the end of follow-up, 196 cases were died, nine cases were lost for follow-up, and 235 patients had recurrent or metastatic cancer. The median DFS and OS were 23 and 61.1 months, respectively.

The median DFS of low and high dNLR TNBC patients was 28.9 and 15.1 months, respectively $(P<0.001$, Figure 1$)$, whereas the median OS of low and high dNLR patients was

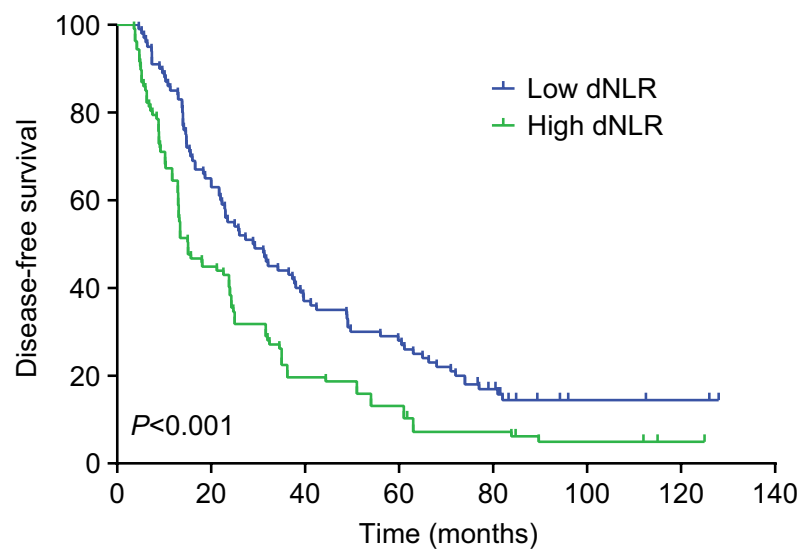

Figure I The disease-free survival in TNBC patients divided by dNLR. Abbreviations: dNLR, derived neutrophil-to-lymphocyte ratio; TNBC, triplenegative breast cancer.

71.2 and 42.3 months, respectively $(P<0.001$; Figure 2$)$. For patients aged $\leq 50$ years, the DFS and OS were higher in low dNLR patients than in high dNLR patients $(25.8$ vs 15.0 months, $P<0.001 ; 68.3$ vs 44.0 months, $P=0.006$; respectively, Figures 3 and 4). For patients with invasive ductal carcinoma, the median DFS of low and high dNLR patients was 29.3 and 14.2 months, respectively $(P<0.001$; Figure 5$)$, whereas the median OS of low and high dNLR patients was 71.6 and 44 months, respectively ( $P=0.002$; Figure 6 ).

Univariate analysis showed that higher tumor stage, lymphovascular invasion, histological grade, lymph node status, and dNLR were related to poor DFS ( $P<0.05$, Table 2$)$. In addition to dNLR, we included confounding factors (age, type of surgery, tumor stage, lymphovascular invasion, histological grade, lymph node status, adjuvant radiotherapy, and chemotherapy) in multivariate analysis. It was showed in the multivariate analysis that increased dNLR was an independent predictor of poor DFS (HR=1.90, 95\% CI: 1.52-2.46, $P=0.007$; Table 2). High dNLR, tumor stage, lymphovascular invasion, histological grade, and lymph node status predicted shorter OS $(P<0.05$, Table 3$)$. Also, increased dNLR was showed an independent predictor of poor OS in multivariate analysis (HR=2.56, 95\% CI: 1.69-3.58, $P=0.001$; Table 3 ). For the clinicopathological parameters, we also found that histological grade and tumor stage were independently related to survival of TNBC patients $(P<0.05$, Tables 2 and 3$)$.

\section{Discussion}

Few studies have reported the correlation between dNLR and the prognosis of TNBC patients, particularly in the Chinese population. To our knowledge, our study included the largest sample size compared with any other studies exploring 


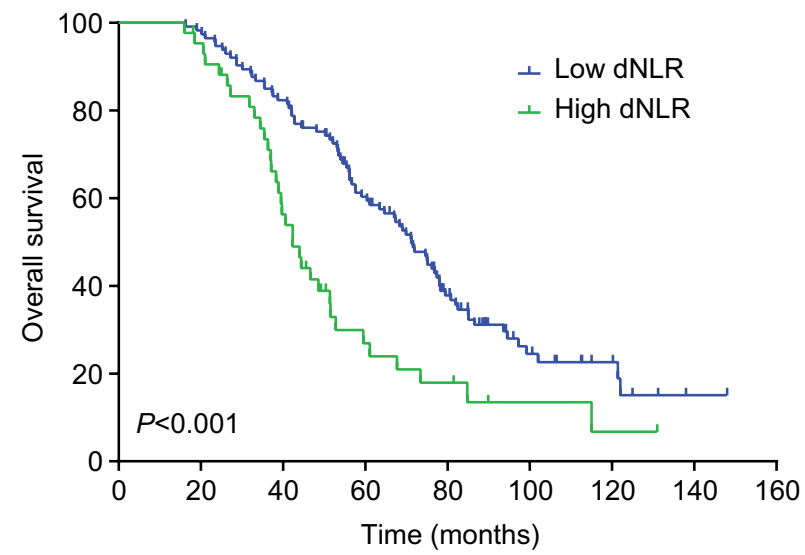

Figure 2 The overall survival in TNBC patients divided by dNLR. Abbreviations: $\mathrm{dNLR}$, derived neutrophil-to-lymphocyte ratio; TNBC, triplenegative breast cancer.

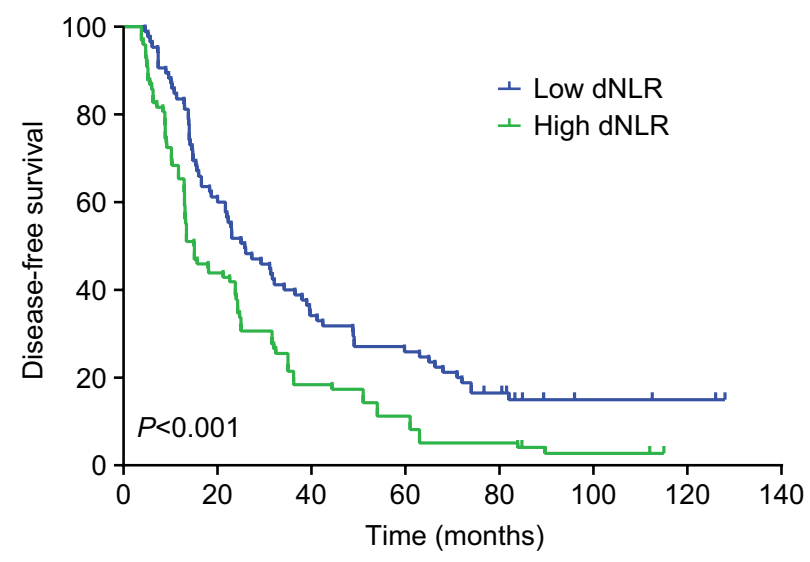

Figure 3 The disease-free survival in TNBC patients aged $\leq 50$ years divided by dNLR.

Abbreviations: $\mathrm{dNLR}$, derived neutrophil-to-lymphocyte ratio; TNBC, triplenegative breast cancer.

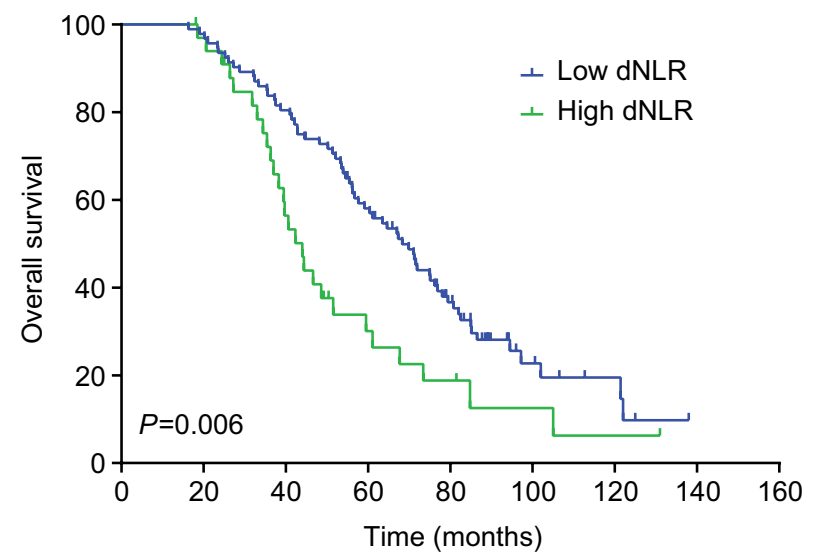

Figure 4 Overall survival of TNBC patients aged $\leq 50$ years divided by $d N L R$. Abbreviations: $\mathrm{dNLR}$, derived neutrophil-to-lymphocyte ratio; TNBC, triplenegative breast cancer.

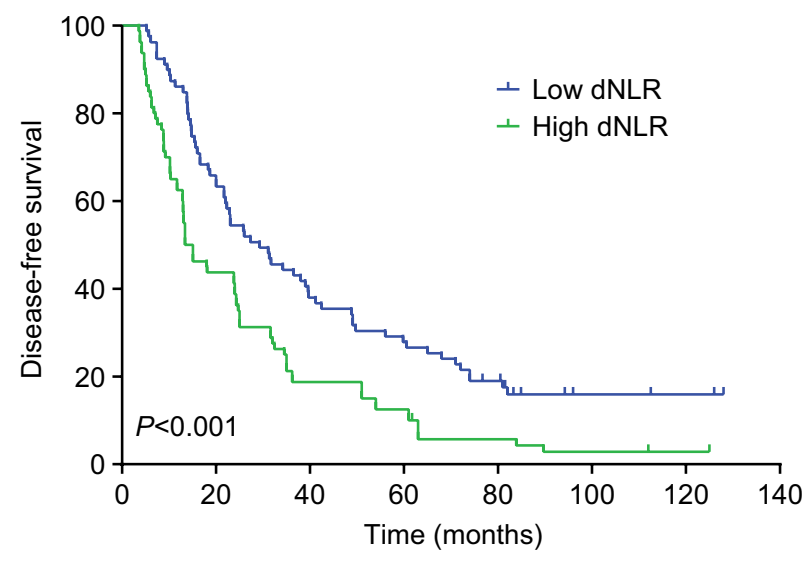

Figure 5 The disease-free survival in TNBC patients with invasive ductal carcinoma divided by dNLR.

Abbreviations: $\mathrm{dNLR}$, derived neutrophil-to-lymphocyte ratio; TNBC, triplenegative breast cancer.

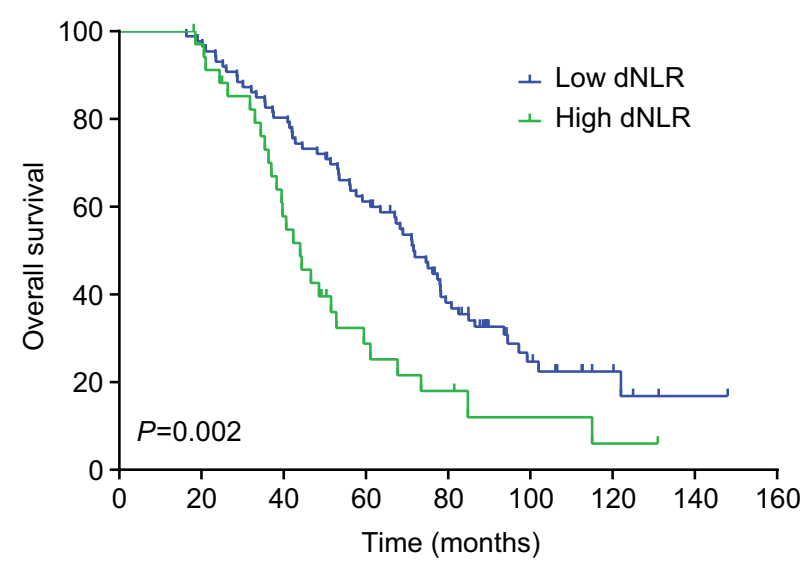

Figure 6 The overall survival in TNBC patients with invasive ductal carcinoma divided by dNLR.

Abbreviations: $\mathrm{dNLR}$, derived neutrophil-to-lymphocyte ratio; TNBC, triplenegative breast cancer.

the value of dNLR in predicting the prognosis of Chinese TNBC patients.

During inflammatory responses, the circulating cytokines and chemokines were released from the increased number of neutrophil and platelet counts. The counts of lymphocyte were declined. ${ }^{28}$ Neutrophils play important roles in tumor expansion, angiogenesis, and metastasis. ${ }^{29}$ Previous studies have demonstrated the association of inflammatory responses with the development, progression, metastasis, and relapse of cancer. ${ }^{30}$ Notably, tumor lymphocyte infiltration appeared to be related to tumor prognosis. ${ }^{6,731}$ Activation status of $\mathrm{T}$ cells was positively associated with the OS in patients with breast cancer. ${ }^{32,33}$ Additionally, it has shown that status of tumor-infiltrating lymphocyte that expressing the pro- 
Table 2 Cox analysis for disease-free survival in $28 \mathrm{I}$ patients with triple-negative breast cancer

\begin{tabular}{|c|c|c|c|c|c|c|}
\hline \multirow[t]{2}{*}{ Variables } & \multicolumn{3}{|c|}{ Univariate analysis } & \multicolumn{3}{|c|}{ Multivariate analysis } \\
\hline & HR & $95 \% \mathrm{Cl}$ & $P$-value & HR & $95 \% \mathrm{Cl}$ & $P$-value \\
\hline \multicolumn{7}{|l|}{ Age (years) } \\
\hline$>50$ vs $\leq 50$ & 0.69 & $0.56-1.09$ & 0.196 & 0.69 & $0.5 I-1.18$ & 0.105 \\
\hline \multicolumn{7}{|l|}{ Type of surgery } \\
\hline Radical mastectomy vs breast conserving surgery & 1.05 & $0.91-1.12$ & 0.244 & 0.92 & $0.84-1.24$ & 0.379 \\
\hline \multicolumn{7}{|l|}{ Tumor stage } \\
\hline T2-4 vs TI & 1.69 & $1.16-2.59$ & 0.006 & 1.50 & $1.31-2.49$ & 0.015 \\
\hline \multicolumn{7}{|l|}{ Lymphovascular invasion } \\
\hline Yes vs no & 1.24 & $1.09-1.76$ & 0.021 & 1.01 & $0.92-1.26$ & 0.152 \\
\hline \multicolumn{7}{|l|}{ Histological grade } \\
\hline III vs I-II & 2.31 & $1.92-3.16$ & $<0.001$ & 2.01 & $1.35-2.59$ & 0.011 \\
\hline \multicolumn{7}{|l|}{ Lymph node status } \\
\hline Yes vs no & 1.94 & $1.46-2.38$ & 0.004 & 1.19 & $1.16-1.86$ & 0.059 \\
\hline \multicolumn{7}{|l|}{ Adjuvant radiotherapy } \\
\hline Yes vs no & 0.77 & $0.69-1.12$ & 0.587 & 0.72 & $0.64-1.07$ & 0.659 \\
\hline \multicolumn{7}{|l|}{ Chemotherapy } \\
\hline Neoadjuvant vs adjuvant & 0.84 & $0.72-1.15$ & 0.489 & 0.77 & $0.69-1.12$ & 0.575 \\
\hline \multicolumn{7}{|l|}{$\mathrm{dNLR}$} \\
\hline$\geq 2.6$ vs $<2.6$ & 2.39 & $1.85-2.59$ & $<0.001$ & 1.90 & $1.52-2.46$ & 0.007 \\
\hline
\end{tabular}

Abbreviation: $\mathrm{dNLR}$, derived neutrophil-to-lymphocyte ratio.

Table 3 Cox analysis for overall survival in 281 patients with triple-negative breast cancer

\begin{tabular}{|c|c|c|c|c|c|c|}
\hline \multirow[t]{2}{*}{ Variables } & \multicolumn{3}{|c|}{ Univariate analysis } & \multicolumn{3}{|c|}{ Multivariate analysis } \\
\hline & HR & $95 \% \mathrm{Cl}$ & $P$-value & HR & $95 \% \mathrm{Cl}$ & $P$-value \\
\hline \multicolumn{7}{|l|}{ Age (years) } \\
\hline$>50$ vs $\leq 50$ & 0.82 & $0.62-1.15$ & 0.354 & 0.77 & $0.5 I-1.26$ & 0.325 \\
\hline \multicolumn{7}{|l|}{ Type of surgery } \\
\hline Radical mastectomy vs breast conserving surgery & 1.10 & $0.82-1.31$ & 0.292 & 0.98 & $0.79-1.06$ & 0.453 \\
\hline \multicolumn{7}{|l|}{ Tumor stage } \\
\hline T2-4 vs TI & 2.43 & $1.79-3.26$ & $<0.001$ & 1.78 & $1.31-2.84$ & 0.002 \\
\hline \multicolumn{7}{|l|}{ Lymphovascular invasion } \\
\hline Yes vs no & 1.50 & $1.18-1.86$ & 0.017 & 1.31 & $1.07-1.62$ & 0.047 \\
\hline \multicolumn{7}{|l|}{ Histological grade } \\
\hline III vs I-II & 3.12 & $2.62-3.51$ & $<0.001$ & 2.30 & $1.75-2.84$ & 0.004 \\
\hline \multicolumn{7}{|l|}{ Lymph node status } \\
\hline Yes vs no & 2.52 & $1.76-3.15$ & 0.002 & 1.86 & $1.46-2.54$ & 0.018 \\
\hline \multicolumn{7}{|l|}{ Adjuvant radiotherapy } \\
\hline Yes vs no & 0.88 & $0.65-1.12$ & 0.837 & 0.80 & $0.62-1.08$ & 0.875 \\
\hline \multicolumn{7}{|l|}{ Chemotherapy } \\
\hline Neoadjuvant vs adjuvant & 0.92 & $0.8 I-1.16$ & 0.860 & 0.83 & $0.68-1.12$ & 0.926 \\
\hline dNLR & & & & & & \\
\hline$\geq 2.6$ vs $<2.6$ & 3.13 & $1.86-4.26$ & $<0.001$ & 2.56 & $1.69-3.58$ & 0.001 \\
\hline
\end{tabular}

Abbreviation: $\mathrm{dNLR}$, derived neutrophil-to-lymphocyte ratio.

grammed cell death 1-ligand 1 was an favorable independent predictor of prognosis for patients with inflammatory breast cancer, suggesting that immune checkpoint immunotherapy should be explored and correlated with prognosis in these patients. ${ }^{34}$

Studies have demonstrated that high dNLR was related to the poor prognosis of multiple cancers. ${ }^{25,35-39}$ Among these, a few studies have explored the role of dNLR in breast cancer, but the results were inconsistency. Proctor et a ${ }^{40}$ found that increased levels of dNLR were related to poor prognosis for patients with breast cancer; however, they did not take into account the clinical stage, tumor histopathological grade, hormone receptor status, and previous treatments of patients, thus making it impossible to assess whether dNLR was associated with prognosis after adjusting other factors. In another study, Dirican et a ${ }^{37}$ analyzed 1,527 cases of breast 
cancer. The results showed a significant association between increased dNLR and the DFS and OS; however, their multivariate analysis did not show an independent prognostic value of dNLR for breast cancer.

In our study, we collected the dNLR 1 week before surgery or neoadjuvant chemotherapy to avoid treatments inducing change in dNLR. Our study provided evidence that dNLR is significantly related to the OS and DFS in TNBC patients. This correlation is still significant after adjusting the patients' age, lymph node metastasis, tumor size, and histopathological grading. Our results suggested that elevated dNLR is independently correlated with high mortality, suggesting that increased dNLR is potentially used as an independent predictor for prognosis in TNBC patients. By releasing ROS, tumor inflammatory mediators, arginase, nitric oxide, and remodeling the extracellular matrix, neutrophils promote tumor development, ${ }^{41,42}$ which may explain our findings. In our study, patients with breast cancer underwent routine blood tests prior to first-line treatment. Consequently, the assessment of dNLR was readily available without any additional costs. Therefore, preoperative dNLR may be used as an indicator to predict the survival of TNBC patients.

However, our study also had some shortcomings. First, there was no external validation used in this study. Second, bias of selecting cases was inevitable in the single-center retrospective study. Despite these limitations, our study still provided strong evidence for a role of dNLR in predicting the prognosis of TNBC patients.

\section{Conclusion}

This study showed that dNLR may be an independent factor for predicting the prognosis of TNBC patients. Patients with high dNLR $(\geq 2.6)$ may have worse survival and may be selected for aggressive therapies, such as high-dose chemotherapy and radiotherapy. Prospective studies with large sample size are still necessary for confirming the prognostic value of dNLR.

\section{Disclosure}

The authors report no conflicts of interest in this work.

\section{References}

1. Kohler BA, Sherman RL, Howlader N, et al. Annual Report to the Nation on the Status of Cancer, 1975-2011, featuring incidence of breast cancer subtypes by race/ethnicity, poverty, and state. J Natl Cancer Inst. 2015;107(6):djv048.

2. Brewster AM, Chavez-Macgregor M, Brown P. Epidemiology, biology, and treatment of triple-negative breast cancer in women of African ancestry. Lancet Oncol. 2014;15(13):e625-e634.
3. Bosch A, Eroles P, Zaragoza R, Viña JR, Lluch A. Triple-negative breast cancer: molecular features, pathogenesis, treatment and current lines of research. Cancer Treat Rev. 2010;36(3):206-215.

4. Sparano JA, Gray RJ, Makower DF, et al. Prospective validation of a 21-gene expression assay in breast cancer. $N$ Engl J Med. 2015;373(21):2005-2014.

5. Ali HR, Provenzano E, Dawson SJ, et al. Association between CD8+ T-cell infiltration and breast cancer survival in 12,439 patients. Ann Oncol. 2014;25(8):1536-1543.

6. Muenst S, Soysal SD, Gao F, Obermann EC, Oertli D, Gillanders WE. The presence of programmed death 1 (PD-1)-positive tumor-infiltrating lymphocytes is associated with poor prognosis in human breast cancer. Breast Cancer Res Treat. 2013;139(3):667-676.

7. Mahmoud SM, Paish EC, Powe DG, et al. An evaluation of the clinical significance of FOXP3+ infiltrating cells in human breast cancer. Breast Cancer Res Treat. 2011;127(1):99-108.

8. Liu C, Huang Z, Wang Q, et al. Usefulness of neutrophil-to-lymphocyte ratio and platelet-to-lymphocyte ratio in hormone-receptor-negative breast cancer. Onco Targets Ther. 2016;9:4653-4660.

9. Koh CH, Bhoo-Pathy N, Ng KL, et al. Utility of pre-treatment neutrophil-lymphocyte ratio and platelet-lymphocyte ratio as prognostic factors in breast cancer. Br J Cancer. 2015;113(1):150-158.

10. Yao M, Liu Y, Jin H, et al. Prognostic value of preoperative inflammatory markers in Chinese patients with breast cancer. Onco Targets Ther. 2014;7:1743-1752.

11. Noh H, Eomm M, Han A. Usefulness of pretreatment neutrophil to lymphocyte ratio in predicting disease-specific survival in breast cancer patients. J Breast Cancer. 2013;16(1):55-59.

12. Yamagishi T, Fujimoto N, Nishi H, et al. Prognostic significance of the lymphocyte-to-monocyte ratio in patients with malignant pleural mesothelioma. Lung Cancer. 2015;90(1):111-117.

13. Spolverato G, Maqsood H, Kim Y, et al. Neutrophil-lymphocyte and platelet-lymphocyte ratio in patients after resection for hepato-pancreatico-biliary malignancies. J Surg Oncol. 2015;111(7):868-874.

14. Liu C, Wu S, Meng X, et al. Predictive value of peripheral regulatory $\mathrm{T}$ cells in non-small cell lung cancer patients undergoing radiotherapy. Oncotarget. 2017;8(26):43427-43438.

15. Peng B, Wang YH, Liu YM, Ma LX. Prognostic significance of the neutrophil to lymphocyte ratio in patients with non-small cell lung cancer: a systemic review and meta-analysis. Int J Clin Exp Med. 2015;8(3): 3098-3106.

16. Ferrucci PF, Gandini S, Battaglia A, et al. Baseline neutrophil-tolymphocyte ratio is associated with outcome of ipilimumab-treated metastatic melanoma patients. Br J Cancer. 2015;112(12):1904-1910.

17. Pinato DJ, Shiner RJ, Seckl MJ, Stebbing J, Sharma R, Mauri FA. Prognostic performance of inflammation-based prognostic indices in primary operable non-small cell lung cancer. Br J Cancer. 2014;110(8):1930-1935.

18. Berardi R, Rinaldi S, Santoni M, et al. Prognostic models to predict survival in patients with advanced non-small cell lung cancer treated with first-line chemo- or targeted therapy. Oncotarget. 2016;7(18):26916-26924.

19. Lolli C, Basso U, Derosa L, et al. Systemic immune-inflammation index predicts the clinical outcome in patients with metastatic renal cell cancer treated with sunitinib. Oncotarget. 2016;7(34):54564-54571.

20. Santoni M, de Giorgi U, Iacovelli R, et al. Pre-treatment neutrophilto-lymphocyte ratio may be associated with the outcome in patients treated with everolimus for metastatic renal cell carcinoma. Br JCancer. 2013;109(7):1755-1759.

21. Song S, Li C, Li S, Gao H, Lan X, Xue Y. Derived neutrophil to lymphocyte ratio and monocyte to lymphocyte ratio may be better biomarkers for predicting overall survival of patients with advanced gastric cancer. Onco Targets Ther. 2017;10:3145-3154.

22. Ferrucci PF, Ascierto PA, Pigozzo J, et al. Baseline neutrophils and derived neutrophil-to-lymphocyte ratio: prognostic relevance in metastatic melanoma patients receiving ipilimumab. Ann Oncol. 2016;27(4):732-738. 
23. Grenader T, Nash S, Plotkin Y, et al. Derived neutrophil lymphocyte ratio may predict benefit from cisplatin in the advanced biliary cancer: the ABC-02 and BT-22 studies. Ann Oncol. 2015;26(9):1910-1916.

24. Deng Q, He B, Liu X, et al. Prognostic value of pre-operative inflammatory response biomarkers in gastric cancer patients and the construction of a predictive model. J Transl Med. 2015;13:66.

25. Absenger G, Szkandera J, Pichler M, et al. A derived neutrophil to lymphocyte ratio predicts clinical outcome in stage II and III colon cancer patients. Br J Cancer. 2013;109(2):395-400.

26. Dalpiaz O, Luef T, Seles M, et al. Critical evaluation of the potential prognostic value of the pretreatment-derived neutrophil-lymphocyte ratio under consideration of $\mathrm{C}$-reactive protein levels in clear cell renal cell carcinoma. Br J Cancer. 2017;116(1):85-90.

27. Russo A, Franchina T, Ricciardi GRR, et al. Baseline neutrophilia, derived neutrophil-to-lymphocyte ratio (dNLR), platelet-to-lymphocyte ratio (PLR), and outcome in non small cell lung cancer (NSCLC) treated with Nivolumab or Docetaxel. J Cell Physiol. 2018;233(10):6337-6343.

28. Diakos CI, Charles KA, Mcmillan DC, Clarke SJ. Cancer-related inflammation and treatment effectiveness. Lancet Oncol. 2014;15(11):e493-e503.

29. de Larco JE, Wuertz BR, Furcht LT. The potential role of neutrophils in promoting the metastatic phenotype of tumors releasing interleukin-8. Clin Cancer Res. 2004;10(15):4895-4900.

30. Grivennikov SI, Greten FR, Karin M. Immunity, inflammation, and cancer. Cell. 2010;140(6):883-899.

31. Tao H, Mimura Y, Aoe K, et al. Prognostic potential of FOXP3 expression in non-small cell lung cancer cells combined with tumor-infiltrating regulatory T cells. Lung Cancer. 2012;75(1):95-101.

32. Shao S, Risch E, Burner D, Lu L, Minev B, Ma W. IFN $\gamma$ enhances cytotoxic efficiency of the cytotoxic T lymphocytes against human glioma cells. Int Immunopharmacol. 2017;47:159-165.

33. Lu L, Bai Y, Wang Z. Elevated T cell activation score is associated with improved survival of breast cancer. Breast Cancer Res Treat. 2017;164(3):689-696.
34. Arias-Pulido H, Cimino-Mathews A, Chaher N, et al. The combined presence of CD20+B cells and PD-L1 + tumor-infiltrating lymphocytes in inflammatory breast cancer is prognostic of improved patient outcome. Breast Cancer Res Treat. 2018;171(2):273-282.

35. Cox S, Hurt C, Grenader T, Mukherjee S, Bridgewater J, Crosby T. The prognostic value of derived neutrophil to lymphocyte ratio in oesophageal cancer treated with definitive chemoradiotherapy. Radiother Oncol. 2017;125(1):154-159.

36. Krenn-Pilko S, Langsenlehner U, Stojakovic T, et al. The elevated preoperative derived neutrophil-to-lymphocyte ratio predicts poor clinical outcome in breast cancer patients. Tumour Biol. 2016;37(1): 361-368.

37. Dirican A, Kucukzeybek BB, Alacacioglu A, et al. Do the derived neutrophil to lymphocyte ratio and the neutrophil to lymphocyte ratio predict prognosis in breast cancer? Int J Clin Oncol. 2015;20(1) 70-81

38. Ying HQ, Deng QW, He BS, et al. The prognostic value of preoperative NLR, d-NLR, PLR and LMR for predicting clinical outcome in surgical colorectal cancer patients. Med Oncol. 2014;31(12):305.

39. Pistelli M, de Lisa M, Ballatore Z, et al. Pre-treatment neutrophil to lymphocyte ratio may be a useful tool in predicting survival in early triple negative breast cancer patients. BMC Cancer. 2015;15:195.

40. Proctor MJ, McMillan DC, Morrison DS, Fletcher CD, Horgan PG, Clarke SJ. A derived neutrophil to lymphocyte ratio predicts survival in patients with cancer. Br J Cancer. 2012;107(4):695-699.

41. Hattar K, Franz K, Ludwig M, et al. Interactions between neutrophils and non-small cell lung cancer cells: enhancement of tumor proliferation and inflammatory mediator synthesis. Cancer Immunol Immunother. 2014;63(12):1297-1306.

42. Houghton AM, Rzymkiewicz DM, Ji H, et al. Neutrophil elastasemediated degradation of IRS-1 accelerates lung tumor growth. Nat Med. 2010;16(2):219-223. 


\section{Supplementary material}

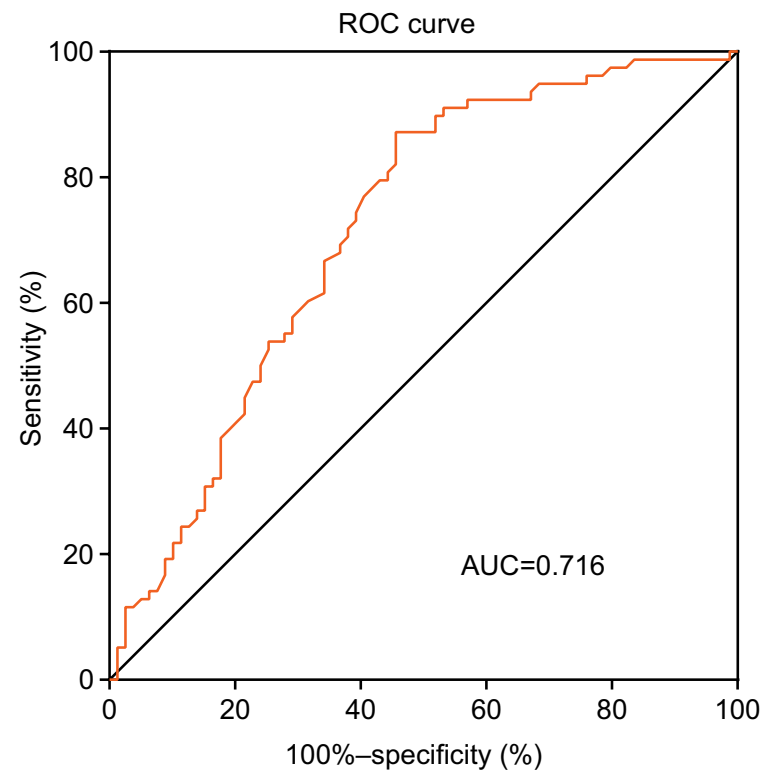

Figure SI ROC curve to distinguish long and short OS by dNLR.

Abbreviations: $d N L R$, derived neutrophil-to-lymphocyte ratio; OS, overall survival; ROC, receiver operating characteristic curve; AUC, area under the curve.

\section{Publish your work in this journal}

Cancer Management and Research is an international, peer-reviewed open access journal focusing on cancer research and the optimal use of preventative and integrated treatment interventions to achieve improved outcomes, enhanced survival and quality of life for the cancer patient. The manuscript management system is completely online and includes

a very quick and fair peer-review system, which is all easy to use. Visit $\mathrm{http}: / / \mathrm{www}$.dovepress.com/testimonials.php to read real quotes from published authors. 